\title{
Clobetasol Propionate Cream Versus Halcinonide Cream in Psoriasis
}

\author{
Charles N. Ellis, M.D., and Eugene J. Van Scott, M.D.
}

From the Clinical Dermatopharmacology Unit, Department of Dermatology, University of Michigan Medical Center and Dermatology Service, Veterans Administration Medical Center, Ann Arbor, Michigan, and the Department of Dermatology, Temple University School of Medicine, Philadelphia, Pennsylvania

$\mathrm{C}^{\prime}$ obetasol propionate, probably the most potent topical corticosteroid yet developed, ${ }^{1}$ has recently been approved for use in the United States. Confirming vasoconstrictor assay predictions of clinical effectiveness, ${ }^{2}$ clobetasol has been shown in doubleblind, multicenter trials ${ }^{3,4}$ to be statistically significantly superior in $0.05 \%$ cream formulation to $0.05 \%$ fluocinonide cream and in $0.05 \%$ ointment formulation to $0.05 \%$ betamethasone dipropionate in an optimized vehicle in the treatment of psoriasis. Clobetasol propionate cream $0.05 \%$ has also been compared with the potent topical corticosteroid cream halcinonide $0.1 \%$ in a recent two-center study. The results of the 2-week randomized double-blind, left-right paired trial (the most accurate comparative measure of topical corticosteroid efficacy in psoriasis) are presented below.

\section{Materials and Methods}

Clobetasol and halcinonide creams were applied twice daily for 2 weeks, in quantities not exceeding $50 \mathrm{~g}$ per week, by 73 patients with moderate-to-severe psoriasis. (Identical blind-labeled tubes of cream were randomly marked for use on patients' right or left sides.) Investigator preference and patient preference were assessed on treatment days 3, 7,

Address for correspondence: Charles N. Ellis, M.D., Dermatology Service, Veterans Administration Medical Center, 2215 Fuller Road, Ann Arbor, MI 48105.

See also page 300 . and 14. Treatment preferences were again assessed 2 weeks after discontinuation of therapy, as was the incidence of relapse by treatment side.

\section{Results}

By investigators' preference, a significant treatment difference in favor of clobetasol became evident as early as the third day and more than doubled by two weeks (Fig. 1). After 2 weeks without therapy, the margin of difference widened further, to $81 \%$ clobetasoltreated sides preferred versus 3\% halcinonide-treated sides preferred. Patient preference showed a similar pattern (Fig. 1). Also, significantly fewer patients showed relapse on the clobetasol side 2 weeks after treatment was stopped (3\% clobetasol versus $63 \%$ halcinonide; $\mathrm{p}<0.001$, McNemar's test).

Both drugs were well tolerated: seven instances of mild adverse cutaneous reactions were reported for clobetasol and nine for halcinonide.

\section{Discussion}

The results of this comparative trial of clobetasol and halcinonide creams demonstrate a superior pharmacologic efficiency of clobetasol in psoriasis as measured by degree, rate, and duration of healing. Similar results have been reported in comparisons of clobetasol and fluocinonide creams $^{3}$ and clobetasol and betamethasone dipropionate ointments ${ }^{4}$ and support the predictive value of the vasoconstrictor assay in determining clinical efficacy.

\section{Drug Names}

betamethasone dipropionate in an optimized vehicle: Diprolene clobetasol: Temovate

fluocinonide: Lidex

halcinonide: Halog 

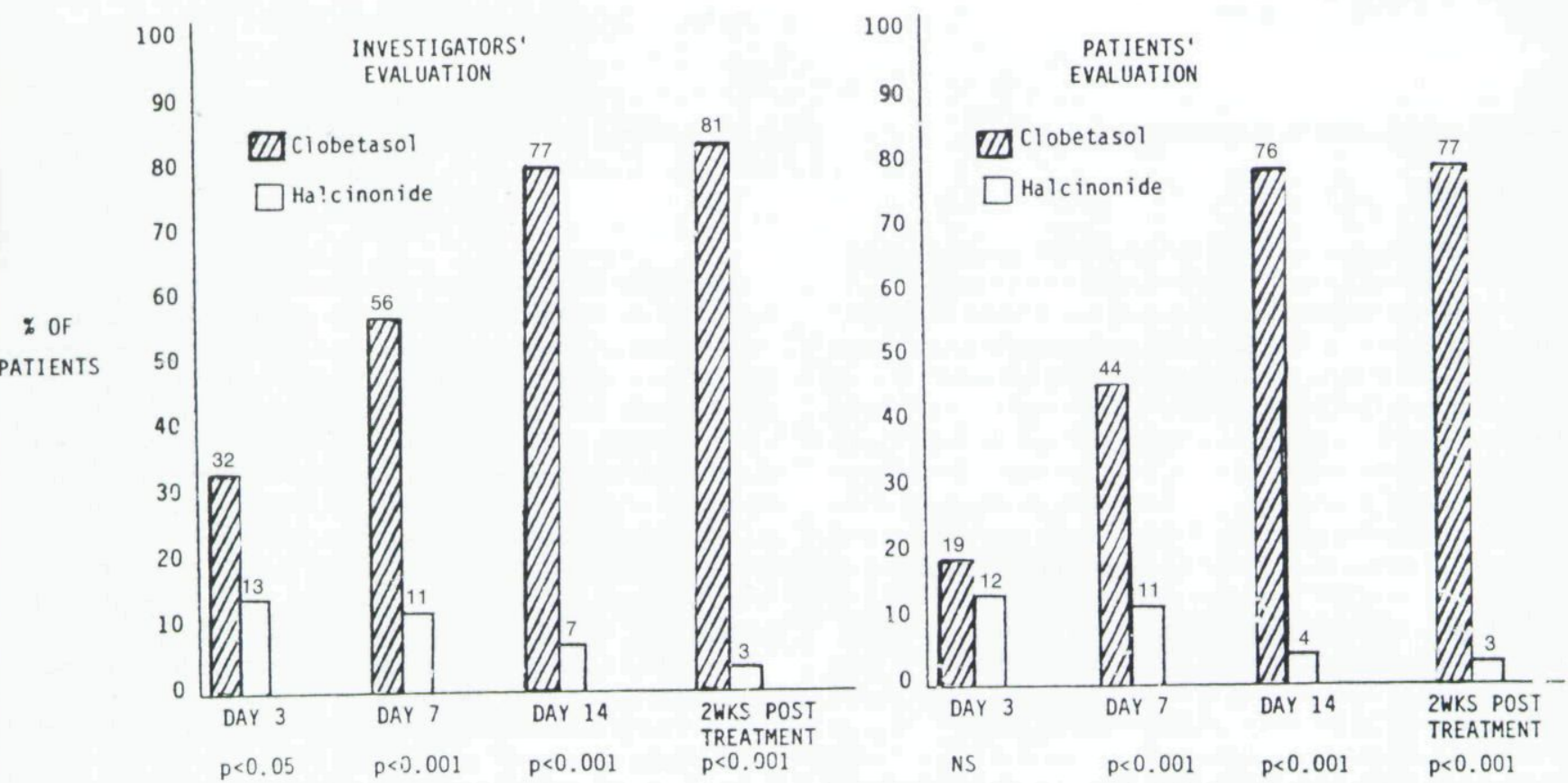

FiG. 1. Percent of treatment sides rated superior according to investigators and patients (preferences compared by sign test).

\section{References}

1. Parish LC, Witkowski JA, Muir JG. Topical corticosteroids. Int J Dermatol. 1985;24:435-436.

2. Cornell RC, Stoughton RB. Correlation of the vasoconstrictor assay and clinical activity in psoriasis. Arch Dermatol. 1985: $121: 63-67$.
3. Jegasothy B, Jacobson C, Levine N, et al. Clobetasol propionate versus fluocinonide creams in psoriasis and eczema. Int J Dermatol. 1985;24:461-465.

4. Jacobson C, Cornell R, Savin R. A comparison of $0.05 \%$ clobetasol propionate ointment and an optimized $0.05 \%$ betamethasone dipropionate ointment in the treatment of psoriasis. Cutis $1986 ; 37: 213-220$.

\section{Leprosy Testing}

Despite the existence of apparently effective chemotherapy since the 1940 s, the prevalence of leprosy in parts of many developing countries remains as high as 10 per 1000 of the population; furthermore, strains that are resistant to the low-cost drug dapsone seem to be increasing in frequency. Much effort is therefore being directed towards understanding the immune response to Mycobacterium leprae and towards development of potentially prophylactic and therapeutic vaccines to combat the disease. Epidemiological evidence suggests that the spread of leprosy is mainly person to person and, since modern chemotherapy rapidly reduces the number of viable organisms spread by an individual, the major source of transmission is likely to be individuals who do not yet have symptoms. This may be particularly true of patients with lepromatous disease, in whom very large numbers of bacilli are present at the time of clinical diagnosis. One of the principal goals of leprosy research is to develop tests that will allow identification and early treatment of such individuals, with the aim of reducing transmission of the disease.-Serological tests for leprosy. Lancet 1986;1:533-535. 
This document is a scanned copy of a printed document. No warranty is given about the accuracy of the copy. Users should refer to the original published version of the material. 